\title{
LINEAR AND NONLINEAR NONLOCAL BOUNDARY VALUE PROBLEMS FOR DIFFERENTIAL-OPERATOR EQUATIONS
}

\author{
RAVI P. AGARWAL, MARTIN BOHNER, AND VELI B. SHAKHMUROV
}

\begin{abstract}
This study focuses on nonlocal boundary value problems (BVP) for linear and nonlinear elliptic differential-operator equations (DOE) that are defined in Banachvalued function spaces. The considered domain is a region with varying bound and depends on a certain parameter. Some conditions that guarantee the maximal $L_{p^{-}}$ regularity and Fredholmness of linear BVP, uniformly with respect to this parameter, are presented. This fact implies that the appropriate differential operator is a generator of an analytic semigroup. Then, by using these results, the existence, uniqueness, and maximal smoothness of solutions of nonlocal BVP for nonlinear DOE are shown. These results are applied to nonlocal boundary value problems for regular elliptic partial differential equations, finite and infinite systems of differential equations on cylindrical domains, in order to obtain the algebraic conditions that guarantee the same properties.
\end{abstract}

\section{Introduction AND Notation}

BVPs for DOE have been studied in detail in $[3,7,12]$. A comprehensive introduction to DOE and historical references may be found in $[2,7]$. The maximal $L_{p}$-regularity for differential operator equations has been discussed in e.g., $[1,5,11]$. The main objective of the present paper is to discuss the maximal regularity of nonlocal BVPs for linear DOEs in Banach-valued $L_{p}$-spaces and the existence and uniqueness of solutions of nonlocal BVPs for nonlinear elliptic DOEs. In this work

(1) BVPs for DOEs are considered in Banach-valued function spaces;

(2) boundary conditions are, in general, nonlocal;

(3) operators used in equations and in boundary conditions are, in general, unbounded;

(4) the considered domain is a region with varying bound and depends on a certain parameter.

The maximal $L_{p}$-regularity and Fredholmness of the linear problems, uniformly with respect to this parameter, are shown, and the existence, uniqueness and maximal smoothness in terms of Sobolev spaces of solutions of nonlinear BVPs are established. These

1991 Mathematics Subject Classification. 34G10, 35J25, 35J70.

Key words and phrases. Boundary value problems, differential-operator equations, maximal $L_{p^{-}}$ regularity, Banach-valued function spaces, operator-valued multipliers, interpolation of Banach spaces, nonlinear DOE, existence. 
results are applied to nonlocal boundary value problems for elliptic and quasi-elliptic partial differential equations and their finite or infinite systems on cylindrical domains with varying bounds.

Let $E$ be a Banach space. Let $L_{p}(\Omega ; E)$ denote the space of strongly measurable $E$ valued functions that are defined on $\Omega$ with the norm

$$
\|f\|_{L_{p}}=\|f\|_{L_{p}(\Omega ; E)}=\left(\int\|f(x)\|_{E}^{p} d x\right)^{\frac{1}{p}}, \quad 1 \leq p<\infty .
$$

By $L_{\mathbf{p}}(\Omega)$ and $W_{\mathbf{p}}^{l}(\Omega), \mathbf{p}=\left(p_{1}, p_{2}\right)$, we will denote a scalar-valued $\mathbf{p}$-summable function space and Sobolev space with mixed norm, respectively [4]. Let $\mathbb{C}$ be the set of complex numbers and

$$
S_{\varphi}=\{\lambda: \lambda \in \mathbb{C},|\arg \lambda-\pi| \leq \pi-\varphi\} \cup\{0\}, \quad 0<\varphi \leq \pi .
$$

A linear operator $A$ is said to be $\varphi$-positive in a Banach space $E$ with bound $M>0$ if $D(A)$ is dense on $E$ and

$$
\left\|(A-\lambda I)^{-1}\right\|_{L(E)} \leq M(1+|\lambda|)^{-1}
$$

with $\lambda \in S_{\varphi}, \varphi \in(0, \pi], I$ is the identity operator in $E$, and $L(E)$ is the space of bounded linear operators in $E$. Sometimes instead of $A+\lambda I$ will be written $A+\lambda$ or $A_{\lambda}$. It is known $[10, \S 1.15 .1]$ that there exist fractional powers $A^{\theta}$ of a positive operator $A$. Let $E\left(A^{\theta}\right)$ denote the space $D\left(A^{\theta}\right)$ with graphical norm.

Let $E_{0} \subset E$ be two Banach spaces. Let $\left(E_{0}, E\right)_{\theta, p}, 0<\theta<1,1 \leq p \leq \infty$, denote interpolation spaces for $\left\{E_{0}, E\right\}$ by the $K$-method [10, 11.3 .1$]$. A function

$$
\Psi \in C^{(l)}\left(\mathbb{R}^{n} \backslash\{0\} ; B\left(E_{0}, E\right)\right), \quad \text { where } \quad l \in \mathbb{N}
$$

is called a multiplier from $L_{p}\left(\mathbb{R}^{n} ; E_{0}\right)$ to $L_{q}\left(\mathbb{R}^{n} ; E\right)$ if there exists a constant $C>0$ such that

$$
\left\|F^{-1} \Psi(\xi) F u\right\|_{L_{q}\left(\mathbb{R}^{n} ; E\right)} \leq C\|u\|_{L_{p}\left(\mathbb{R}^{n} ; E_{0}\right)}
$$

for all $u \in L_{p}\left(\mathbb{R}^{n} ; E_{0}\right)$, where $\hat{u}=F u$ is the Fourier transformation of $u$. We denote the set of all multipliers from $L_{p}\left(\mathbb{R}^{n} ; E_{0}\right)$ to $L_{q}\left(\mathbb{R}^{n} ; E\right)$ by $M_{p}^{q}\left(E_{0}, E\right)$.

A set $K \subset B\left(E_{0}, E\right)$ is called $R$-bounded if there is a constant $C$ such that for all $T_{1}, T_{2}, \ldots, T_{m} \in K$ and $u_{1}, u_{2}, \ldots, u_{m} \in E_{0}, m \in \mathbb{N}$,

$$
\int_{0}^{1}\left\|\sum_{j=1}^{m} r_{j}(y) T_{j} u_{j}\right\|_{E} d y \leq C \int_{0}^{1}\left\|\sum_{j=1}^{m} r_{j}(y) u_{j}\right\|_{E_{0}} d y,
$$

where $\left\{r_{j}\right\}$ is a sequence of independent symmetric $[-1,1]$-valued random variables on $[0,1]$ (see [5]). Let

$$
U_{n}=\left\{\beta=\left(\beta_{1}, \beta_{2}, \ldots, \beta_{n}\right):|\beta| \leq n\right\}, \quad \xi^{\beta}=\xi_{1}^{\beta_{1}} \xi_{2}^{\beta_{2}} \cdots \xi_{n}^{\beta_{n}} .
$$


Definition 1.1. A Banach space $E$ is said to be a space satisfying the multiplier condition with respect to $p \in(1, \infty)$ when for every $\Psi \in C^{(n)}\left(\mathbb{R}^{n} \backslash\{0\} ; B(E)\right)$ if the set

$$
\left\{\xi^{\beta} D_{\xi}^{\beta} \Psi(\xi): \xi \in \mathbb{R}^{n} \backslash\{0\}, \beta \in U_{n}\right\}
$$

is $R$-bounded, then $\Psi \in M_{p}^{p}(E)$. Moreover, if $K$ is a subset of $C^{(n)}\left(\mathbb{R}^{n} \backslash\{0\} ; B(E)\right)$ such that the set (1.1) is uniformly $R$-bounded for $\Psi \in K$, then $K$ is called a uniform collection of multipliers.

Remark 1.2. If $E$ is a UMD space and $n=1$ or if $E$ is a UMD space with property $(\alpha)$ [6] and $n>1$, then $E$ satisfies the multiplier condition (see also [5, 11]).

Definition 1.3. The positive operator $A$ is said to be $R$-positive in the Banach space $E$ if there exists $\varphi \in(0, \pi]$ such that the set

$$
L_{A}=\left\{(1+|\xi|)(A-\xi I)^{-1}: \xi \in S_{\varphi}\right\}
$$

is $R$-bounded.

Note that in Hilbert spaces every norm-bounded set is $R$-bounded.

Let $\sigma_{\infty}(E)$ denote the space of compact operators acting in $E$. Let us consider the space $W_{p}^{l}\left(\Omega ; E_{0}, E\right), \Omega \subset \mathbb{R}^{n}, E_{0} \subset E, l=\left(l_{1}, \ldots, l_{n}\right)$, that consists of functions $u \in L_{p}\left(\Omega ; E_{0}\right)$ that have the generalized derivatives $D_{k}^{l_{k}} u=\frac{\partial^{l_{k}}}{\partial x_{k}^{l_{k}}} u \in L_{p}(\Omega ; E)$ with the norm

$$
\|u\|_{W_{p}^{l}}=\|u\|_{W_{p}^{l}\left(\Omega ; E_{0}, E\right)}=\|u\|_{L_{p}\left(\Omega ; E_{0}\right)}+\sum_{k=1}^{n}\left\|D_{k}^{l_{k}} u\right\|_{L_{p}(\Omega ; E)}<\infty .
$$

For $n=1, l_{1}=l, \Omega=(a, b) \subset \mathbb{R}$, the space $W_{p}^{l}\left(\Omega ; E_{0}, E\right)$ will be denoted by $W_{p}^{l}\left(a, b ; E_{0}, E\right)$.

\section{Statement of the Problem}

Let $b=b(t)$ be a positive continuous function on $[c, d]$. Consider a nonlocal linear BVP on $(0, b)$

$$
\begin{gathered}
L u:=-u^{\prime \prime}(x)+A u(x)+B_{1}(x) u^{\prime}(x)+B_{2}(x) u(x)=f(x), \quad x \in(0, b(t)), \\
L_{t k} u:=\alpha_{k} u^{\left(m_{k}\right)}(0)+\beta_{k} u^{\left(m_{k}\right)}(b)+\sum_{j=1}^{N_{k}} \delta_{k j} u^{\left(m_{k}\right)}\left(x_{t k j}\right)+\sum_{j=1}^{M_{k}} T_{k j} u\left(x_{t k j 0}\right)=f_{k}, \\
k \in\{1,2\},
\end{gathered}
$$

where $f_{k} \in E_{k}=(E(A), E)_{\theta_{k}, p}, \theta_{k}=\frac{m_{k}}{2}+\frac{1}{2 p}, p \in(1, \infty), m_{k} \in\{0,1\} ; \alpha_{k}, \beta_{k}, \delta_{k j}$ are, in general, complex-valued continuous functions of $t, x_{t k j} \in(0, b), x_{t k j 0} \in[0, b], x_{t k j}$ and $x_{t k j 0}$ are continuous in $t ; A, B_{k}(x)$ for $x \in[0, b]$, and $T_{k j}=T_{k j}(t)$ are, generally speaking, unbounded operators in $E$. 
Let us also consider the nonlinear BVP

$$
\begin{gathered}
L u:=-u^{\prime \prime}(x)+A\left(x, u, u^{\prime}\right) u+\lambda u=F\left(x, u, u^{\prime}\right), \quad x \in(0, a), \\
L_{k} u:=\alpha_{k} u^{\left(m_{k}\right)}(0)+\beta_{k} u^{\left(m_{k}\right)}(a)+\sum_{j=1}^{N_{k}} \delta_{k j} u^{\left(m_{k}\right)}\left(x_{k j}\right)=f_{k}, \quad k \in\{1,2\},
\end{gathered}
$$

where $\alpha_{k}, \beta_{k}, \delta_{k j}$ are, in general, complex numbers and $0<a \leq a_{0}, x_{k j} \in(0, a), f_{k} \in E_{k}$, and $A=A(0,0,0)$ is a positive operator in $E$.

The problem (2.1)-(2.2) is said to be maximal $L_{p}$-regular if the problem (2.1) $-(2.2)$ for all $f \in L_{p}(0, b ; E)$ has a unique solution $u \in W_{p}^{2}(0, b ; E(A), E)$ satisfying

$$
\|A u\|_{L_{p}(0, b ; E)}+\left\|u^{\prime \prime}\right\|_{L_{p}(0, b ; E)} \leq\|f\|_{L_{p}(0, b ; E)} .
$$

\section{Background Material}

From $[1,9]$ we obtain the following background material:

Theorem 3.1. Suppose

(1) $E$ is a Banach space satisfying the multiplier condition;

(2) $\alpha=\left(\alpha_{1}, \alpha_{2}, \ldots, \alpha_{n}\right), l=\left(l_{1}, l_{2}, \ldots, l_{n}\right)$ are $n$-tuples of nonnegative integers such that

$$
\kappa=|\alpha: l|=\sum_{k=1}^{n} \frac{\alpha_{k}}{l_{k}} \leq 1, \quad 0 \leq \mu \leq 1-\kappa ;
$$

(3) $A$ is an $R$-positive operator in $E$ for $\varphi \in(0, \pi]$;

(4) $\Omega \subset \mathbb{R}^{n}$ is a region such that there exists a bounded linear extension operator acting from $L_{p}(\Omega ; E)$ to $L_{p}\left(\mathbb{R}^{n} ; E\right)$ and also from $W_{p}^{l}(\Omega ; E(A), E)$ to $W_{p}^{l}\left(\mathbb{R}^{n} ; E(A), E\right)$.

Then the embedding

$$
D^{\alpha} W_{p}^{l}(\Omega ; E(A), E) \subset L_{p}\left(\Omega ; E\left(A^{1-\kappa-\mu}\right)\right)
$$

is continuous and there exists a positive constant $C_{\mu}$ such that

$$
\left\|D^{\alpha} u\right\|_{L_{p}\left(\Omega ; E\left(A^{1-\kappa-\mu}\right)\right)} \leq C_{\mu}\left[h^{\mu}\|u\|_{W_{p}^{l}(\Omega ; E(A), E)}+h^{-(1-\mu)}\|u\|_{L_{p}(\Omega ; E)}\right]
$$

for all $u \in W_{p}^{l}(\Omega ; E(A), E)$ and $0<h \leq h_{0}<\infty$.

Theorem 3.2. Suppose all conditions of Theorem 3.1 are satisfied and suppose $\Omega$ is a bounded region in $\mathbb{R}^{n}$ and $A^{-1} \in \sigma_{\infty}(E)$. Then for $0<\mu \leq 1-\kappa$ the embedding (3.1) is compact.

Theorem 3.3. Suppose all conditions of Theorem 3.1 are satisfied. Then the embedding

$$
D^{\alpha} W_{p}^{l}(\Omega ; E(A), E) \subset L_{p}\left(\Omega ;(E(A), E)_{\kappa, 1}\right)
$$

is continuous and there exists a positive constant $C$ such that

$$
\left\|D^{\alpha} u\right\|_{L_{p}\left(\Omega ;(E(A), E)_{\kappa, 1}\right)} \leq C\|u\|_{W_{p}^{l}(\Omega ; E(A), E)}
$$

for all $u \in W_{p}^{l}(\Omega ; E(A), E)$. 
Theorem 3.4. Let $E$ be a Banach space and $A$ be a positive operator in $E$ of type $\varphi$. Let $m \in \mathbb{N}, 1 \leq p<\infty$, and $\frac{1}{2 p}<\alpha<m+\frac{1}{2 p}$. Let $0 \leq \gamma<2 p \alpha-1$. Then for $\lambda \in S(\varphi)$, the operator $-A_{\lambda}^{1 / 2}$ generates a semigroup $e^{-A_{\lambda}^{1 / 2} x}$, which is holomorphic for $x>0$ and strongly continuous for $x \geq 0$. Moreover, there exists a constant $C>0$ such that

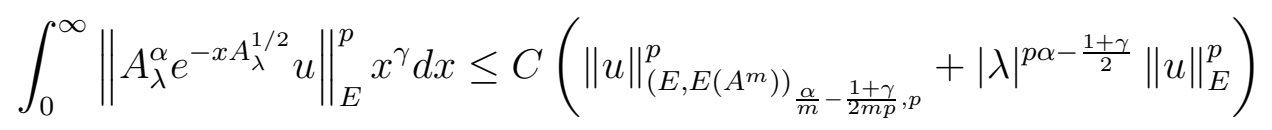

for every $u \in\left(E, E\left(A^{m}\right)\right)_{\frac{\alpha}{m}-\frac{1+\gamma}{2 p m}, p}$ and $\lambda \in S(\varphi)$.

By using a similar technique as in [8] (or $[10, \S 1.8 .1]$ ) we obtain the following.

Theorem 3.5. Let $l, s \in \mathbb{N}_{0}$ with $0 \leq s \leq l-1, \theta=\frac{p s+1}{p l}, x_{0} \in[0, b], 0<h \leq h_{0}$. Then the mapping $u \rightarrow u^{(s)}\left(x_{0}\right)$ is continuous from $W_{p}^{l}\left(0, b ; E_{0}, E\right)$ onto $\left(E_{0}, E\right)_{\theta, p}$,

$$
\left\|u^{(s)}\left(x_{0}\right)\right\|_{\left(E_{0}, E\right)_{\theta, p}} \leq C\|u\|_{W_{p}^{l}\left(0, b ; E_{0}, E\right)},
$$

and

$$
\left\|u^{(s)}\left(x_{0}\right)\right\|_{E} \leq C\left[h^{1-\theta}\left\|u^{(l)}\right\|_{L_{p}(0, b ; E)}+h^{-\theta}\|u\|_{L_{p}(0, b ; E)}\right] .
$$

\section{Homogeneous Equations}

Let us first consider the problem

$$
\begin{gathered}
L_{0}(\lambda) u:=-u^{\prime \prime}(x)+(A+\lambda) u(x)=0 \\
L_{t k 0} u:=\alpha_{k} u^{\left(m_{k}\right)}(0)+\beta_{k} u^{\left(m_{k}\right)}(b)+\sum_{j=1}^{N_{k}} \delta_{k j} u^{\left(m_{k}\right)}\left(x_{t k j}\right)=f_{k}, \quad k \in\{1,2\},
\end{gathered}
$$

Theorem 4.1. Let the following conditions be satisfied:

(1) $A$ is a $\varphi$-positive operator in a Banach space $E$ for $\varphi \in(0, \pi]$;

(2) $\theta=\theta(t)=(-1)^{m_{1}} \alpha_{1} \beta_{2}-(-1)^{m_{2}} \alpha_{2} \beta_{1} \neq 0$ and $\theta_{k}=m_{k} / 2+1 /(2 p)$ for $k \in\{1,2\}$.

Then the problem (4.1)-(4.2) for $f_{k} \in E_{k},|\arg \lambda| \leq \pi-\varphi$ and sufficiently large $|\lambda|$ has a unique solution that belongs to the space $W_{p}^{2}(0, b ; E(A), E)$, and coercive uniformity with respect to the parameters $t$ and $\lambda$ holds, i.e.,

$$
\sum_{i=0}^{2}|\lambda|^{1-\frac{i}{2}}\left\|u^{(i)}\right\|_{L_{p}}+\|A u\|_{L_{p}} \leq M \sum_{k=1}^{2}\left(\left\|f_{k}\right\|_{E_{k}}+|\lambda|^{1-\theta_{k}}\left\|f_{k}\right\|_{E}\right)
$$

is satisfied for the solution of the problem (4.1)-(4.2).

Proof. By condition (1) and by virtue of $[10, \S 1.14]$ for $|\arg \lambda| \leq \pi-\varphi$, there exists the holomorphic (for $x>0$ ) and strongly continuous (for $x \geq 0$ ) $\operatorname{semigroup} e^{-x A_{\lambda}^{1 / 2}}$. In a similar way as in [12, Lemma 5.3.2/1], we obtain that an arbitrary solution of the equation (4.1), for $|\arg \lambda| \leq \pi-\varphi$, belonging to the space $W_{p}^{2}(0, b ; E(A), E)$, has the form

$$
u(x)=V_{\lambda 1} g_{1}+V_{\lambda 2} g_{2},
$$


where

$$
g_{k} \in(E(A), E)_{\frac{1}{2 p}, p}, \quad V_{\lambda 1}=e^{-x A_{\lambda}^{1 / 2}}, \quad V_{\lambda 2}=e^{-(b-x) A_{\lambda}^{1 / 2}} .
$$

Now taking into account the boundary conditions (4.2), we obtain a representation of the solution of the problem (4.1)-(4.2) as

$$
u(x)=Q(\lambda, t)\left[\sum_{k=1}^{2} \sum_{i=1}^{2} C_{k i}(\lambda, t) V_{\lambda k}(x) A_{\lambda}^{-\nu_{k}} f_{i}\right],
$$

where $Q(\lambda, t)$ is a uniformly (with respect to $\lambda$ and $t$ ) bounded operator in $E$. By virtue of the properties of holomorphic semigroups [10, $\S 1.14]$ and by using Theorem 3.4, from (4.4) we obtain the estimate (4.3).

\section{Nonhomogenous Equations}

Now consider a boundary value problem for nonhomogenous equations with parameters on the region $(0, b)$ :

$$
\begin{gathered}
L_{0}(\lambda) u:=-u^{\prime \prime}(x)+(A+\lambda I) u(x)=f(x), \\
L_{t k 0} u:=\alpha_{k} u^{\left(m_{k}\right)}(0)+\beta_{k} u^{\left(m_{k}\right)}(b)+\sum_{j=1}^{N_{k}} \delta_{k j} u^{\left(m_{k}\right)}\left(x_{t k j}\right)=f_{k}, \quad k \in\{1,2\} .
\end{gathered}
$$

Theorem 5.1. Let all conditions of Theorem 4.1 be satisfied, let $E$ be a Banach space satisfying the multiplier condition, and let $A$ be an $R$-positive operator in $E$ for $\varphi \in$ $(0, \pi]$. Then the operator $u \rightarrow D_{0}(\lambda, t) u:=\left\{L_{0}(\lambda) u, L_{t 10} u, L_{t 20} u\right\}$ for $|\arg \lambda| \leq \pi-\varphi$, $0<\varphi \leq \pi$, and sufficiently large $|\lambda|$, is an isomorphism from $W_{p}^{2}(0, b ; E(A), E)$ onto $L_{p}(0, b ; E) \times E_{1} \times E_{2}$. Moreover, coercive uniformity with respect to the parameters $\lambda$ and $t$ holds, i.e.,

$$
\sum_{j=0}^{2}|\lambda|^{1-\frac{j}{2}}\left\|u^{(j)}\right\|_{L_{p}}+\|A u\|_{L_{p}} \leq C\left[\|f\|_{L_{p}}+\sum_{k=1}^{2}\left(\left\|f_{k}\right\|_{E_{k}}+|\lambda|^{1-\theta_{k}}\left\|f_{k}\right\|_{E}\right)\right]
$$

is satisfied for the solution of the problem (5.1)-(5.2).

Proof. We have proved the uniqueness of the solution of problem (5.1)-(5.2) in Theorem 4.1. Let

$$
\bar{f}_{t}(x)=\left\{\begin{array}{lll}
f(x) & \text { if } & x \in[0, b(t)] \\
0 & \text { if } & x \notin[0, b(t)] .
\end{array}\right.
$$

We now show that a solution of the problem (5.1)-(5.2) which belongs to the space $W_{p}^{2}(0, b ; E(A), E)$ can be represented as a sum $v(x)=u_{1}(x)+u_{2}(x)$, where $u_{1}$ is the restriction on $[0, b]$ of the solution $u$ of the equation

$$
L_{0}(\lambda) u=\bar{f}_{t}(x), \quad x \in \mathbb{R}=(-\infty, \infty)
$$


and $u_{2}$ is a solution of the problem

$$
L_{0}(\lambda) u=0, \quad L_{t k 0} u=f_{k}-L_{t k 0} u_{1} .
$$

A solution of equation (5.4) is given by the formula

$$
u(x)=F^{-1} L_{0}^{-1}(\lambda, t, \xi) F \bar{f}_{t}=\frac{1}{2 \pi} \int_{-\infty}^{\infty} e^{i \xi x} L_{0}^{-1}(\lambda, t, \xi)\left(F \bar{f}_{t}\right)(\xi) d \xi,
$$

where $F \bar{f}$ is the Fourier transform of the function $\bar{f}$, and

$$
L_{0}(\lambda, \xi)=\left(\xi^{2}+\lambda\right) I+A .
$$

Using $R$-positivity of $A$, we show that the operator functions

$$
\Psi_{\lambda}(\xi)=A L_{0}^{-1}(\lambda, \xi) \quad \text { and } \quad \Psi_{\lambda, j}(\xi)=|\lambda|^{1-\frac{j}{2}} \xi^{j} L_{0}^{-1}(\lambda, \xi)
$$

are Fourier multipliers in $L_{p}(\mathbb{R} ; E)$. This implies that the problem (5.4) has a solution $u \in W_{p}^{2}(\mathbb{R} ; E(A), E)$ and

$$
\sum_{j=0}^{2}|\lambda|^{1-\frac{j}{2}}\left\|u^{(j)}\right\|_{L_{p}}+\|A u\|_{L_{p}} \leq C\|f\|_{L_{p}} .
$$

Moreover, by using Theorem 4.1 and (the trace) Theorem 3.5, we obtain that the problem (5.5) has a solution $u_{2} \in W_{p}^{2}(0, b ; E(A), E)$ with the estimate (4.3). Then the estimates (4.3) and (5.6) imply (5.3).

Remark 5.2. Let $O$ be a realization operator of the problem $(2.1)-(2.2)$ in $L_{p}(0, b ; E)$, i.e.,

$$
\begin{gathered}
D(O)=\left\{W_{p}^{2}(0, b ; E(A), E), L_{1} u=0, L_{2} u=0\right\} \\
O u=-u^{\prime \prime}(x)+A u(x), \quad x \in(0, b(t)) .
\end{gathered}
$$

By Theorem 5.1, the differential operator $O$ has a resolvent $(O-\lambda I)^{-1}$ for $\lambda \in S(\varphi)$, $0<\varphi \leq \pi$, and uniformity with respect to the parameter $t$

$$
\sum_{i=0}^{2}|\lambda|^{1-\frac{i}{2}}\left\|D^{i}(O-\lambda I)^{-1}\right\|_{B\left(L_{p}(0, b ; E)\right)}+\left\|A(O-\lambda I)^{-1}\right\|_{B\left(L_{p}(0, b ; E)\right)} \leq C
$$

holds. This estimate implies that the operator $O$ is a generator of an analytic semigroup in $L_{p}(0, b ; E)$ for $\varphi<\frac{\pi}{2}$.

\section{Coerciveness on the Space Variable and Fredholmness}

Consider the problem (2.1)-(2.2).

Theorem 6.1. Let the following conditions be satisfied:

(1) $E$ is a Banach space satisfying the multiplier condition and $A$ is an R-positive operator in $E$ for $\varphi=\pi$ and $A^{-1} \in \sigma_{\infty}(E)$;

(2) $\theta(t)=(-1)^{m_{1}} \alpha_{1} \beta_{2}-(-1)^{m_{2}} \alpha_{2} \beta_{1} \neq 0, \theta_{k}=\frac{m_{k}}{2}+\frac{1}{2 p}$; 
(3) for any $\varepsilon>0$ there is $C(\varepsilon)$ such that for almost all $x \in[0, b]$,

$$
\begin{array}{cl}
\left\|B_{1}(x) u\right\| \leq \varepsilon\|u\|_{(E(A), E)_{1 / 2,1}}+C(\varepsilon)\|u\|, & u \in(E(A), E)_{1 / 2,1}, \\
\left\|B_{2}(x) u\right\| \leq \varepsilon\|A u\|+C(\varepsilon)\|u\|, & u \in D(A),
\end{array}
$$

where the functions $B_{1}(x) u$ for $u \in(E(A), E)_{1 / 2,1}$ and $B_{2}(x) u$ for $u \in D(A)$ are measurable on $[0,1]$ in $E$;

(4) if $m_{k}=0$, then $T_{k j}=0$; if $m_{k}=1$, then for any $\varepsilon>0$ there is $C(\varepsilon)$ such that for $u \in(E(A), E)_{\frac{1}{2 p}, p}, p>1$,

$$
\left\|T_{k j} u\right\|_{(E(A), E)_{\frac{1}{2}+\frac{1}{2 p}, p}} \leq \varepsilon\|u\|_{(E(A), E)_{\frac{1}{2 p}, p}}+C(\varepsilon)\|u\| .
$$

Then for all $u \in W_{p}^{2}(0, b ; E(A), E)$, coercive uniformity with respect to the parameters $t$ and $\lambda$ holds, i.e.,

$$
\sum_{i=0}^{2}|\lambda|^{1-\frac{i}{2}}\left\|u^{(i)}\right\|_{L_{p}} \leq C\left[\|L u\|_{L_{p}}+\sum_{k=1}^{2}\left\|L_{t k} u\right\|_{E_{k}}+\|u\|_{L_{p}}\right]
$$

is satisfied for the solution of the problem (2.1)-(2.2). Moreover, the operator $u \rightarrow$ $D(t) u=\left\{L u, L_{t 1} u, L_{t 2} u\right\}$ from $W_{p}^{2}(0, b ; E(A), E)$ into $L_{p}(0,1 ; E) \times E_{1} \times E_{2}$ is bounded and Fredholm.

Proof. Assume that the condition (1) is satisfied for $\arg \lambda=\pi$. The general case is reduced to the latter if the operator $A+\lambda_{0} I$, for some sufficiently large $\lambda_{0}>0$, is considered instead of the operator $A$, and the operator $B_{2}(x)-\lambda_{0} I$ is considered instead of the operator $B_{2}(x)$. Let $u \in W_{p}^{2}(0, b ; E(A), E)$ be a solution of the problem $(2.1)-(2.2)$. Then $u$ is a solution of the problem

$$
\begin{gathered}
-\frac{d^{2}}{d x^{2}} u(x)+(A+\lambda I) u(x)=f(x)+\lambda u(x)-B_{1}(x) \frac{d}{d x} u(x)-B_{2}(x) u(x), \\
L_{t k 0} u=f_{k}-\sum_{j=1}^{M_{k}} T_{k j} u\left(x_{k j 0}\right), \quad k \in\{1,2\}
\end{gathered}
$$

where $L_{t k 0}$ are defined in (4.2). By Theorem 5.1, for sufficiently large $\lambda_{0}>0$, we have

$$
\begin{aligned}
\sum_{i=0}^{2}|\lambda|^{1-\frac{i}{2}}\left\|u^{(i)}\right\|_{L_{p}}+\|A u\|_{L_{p}} \leq C\left[\| f+\lambda_{0} u-\right. & B_{1} u^{(1)}-B_{2} u \|_{L_{p}} \\
& \left.+\sum_{k=1}^{2}\left\|f_{k}-\sum_{j=1}^{M_{k}} T_{k j} u\left(x_{t k j 0}\right)\right\|_{E_{k}}\right] .
\end{aligned}
$$

By Theorem 3.1, Theorem 3.3, and condition (3), for all $u \in W_{p}^{2}(0, b ; E(A), E)$ we have

$$
\left\|B_{1} u^{(1)}\right\|_{L_{p}} \leq \varepsilon\left\|u^{(1)}\right\|_{W_{p}^{1}\left(0, b ;(E(A), E)_{1 / 2,1}, E\right)}+C(\varepsilon)\left\|u^{(1)}\right\|_{L_{p}},
$$




$$
\left\|B_{2} u\right\|_{L_{p}} \leq \varepsilon\|u\|_{W_{p}^{1}(0, b ; E(A), E)}+C(\varepsilon)\|u\|_{L_{p}}, \quad \varepsilon>0 .
$$

From these two inequalities, we obtain for $u \in W_{p}^{2}(0, b ; E(A) ; E)$ by using Theorem 3.1

$$
\max \left\{\left\|B_{1} u^{(1)}\right\|_{L_{p}},\left\|B_{2} u\right\|_{L_{p}}\right\} \leq \varepsilon\|u\|_{W_{p}^{2}(0, b ; E(A), E)}+C(\varepsilon)\|u\|_{L_{p}} .
$$

By virtue of Theorem 3.5, the operator $u \rightarrow u\left(x_{0}\right)$ from $W_{p}^{2}(0, b ; E(A), E)$ into $(E(A), E)_{\frac{1}{2 p}, p}$ is bounded and

$$
\left\|u\left(x_{0}\right)\right\|_{(E(A), E)_{\frac{1}{2 p}, p}} \leq C\|u\|_{W_{p}^{2}(0, b ; E(A), E)} .
$$

Consequently, from condition (4) and the estimate (6.4), it follows for all $\varepsilon>0$ and $u \in W_{p}^{2}(0, b ; E(A), E)$ that

$$
\left\|T_{k j} u\left(x_{k j 0}\right)\right\|_{E_{k}} \leq \varepsilon\|u\|_{W_{p}^{2}(0, b ; E(A), E)}+C(\varepsilon)\|u\|_{L_{p}} .
$$

Using the estimates (6.2), (6.3), and (6.5), we get (6.1).

Next, the operator $D$ can be rewritten in the form

$$
D=D_{0}\left(\lambda_{0}, t\right)+L_{1}, \quad \text { where } \quad D_{0}\left(\lambda_{0}, t\right) u=\left\{L_{0}(\lambda) u, L_{t 10}, L_{t 20}\right\},
$$

using the notation from (4.1)-(4.2), and

$$
D_{1}\left(\lambda_{0}, t\right) u=\left\{-\lambda_{0} u(x)+B_{1}(x) u^{(1)}(x)+B_{2}(x) u(x), \sum_{j=1}^{M_{1}} T_{1 j} u\left(x_{t 1 j}\right), \sum_{j=1}^{M_{2}} T_{2 j} u\left(x_{t 2 j}\right),\right\} .
$$

We can conclude from the first part of this theorem that the operator $D_{0}\left(\lambda_{0}, t\right)$ from $W_{p}^{2}$ onto $L_{p} \times E_{1} \times E_{2}$ has an inverse. From the estimate (6.1) and in view of Theorem 3.1 and Theorem 3.2, it follows that the operator $D_{1}$ from $W_{p}^{2}$ into $L_{p} \times E_{1} \times E_{2}$ is compact. Then by the perturbation theory for linear operators, it follows that the operator $D(t)$ from $W_{p}^{2}$ into $L_{p} \times E_{1} \times E_{2}$ is a Fredholm operator.

\section{NONLinear BVPs FOR DOEs}

Consider the nonlinear BVP (2.3)-(2.4). Let

$$
\begin{gathered}
E_{k}=(E(A), E)_{\eta_{k}, p}, \quad \eta_{k}=\frac{k}{2}+\frac{1}{2 p}, \quad k \in\{0,1\}, \quad F_{0}=E_{0} \times E_{1} \\
X=L_{p}(0, a ; E), \quad Y=W_{p}^{2}((0, a) ; E(A), E), \quad 0<a \leq a_{0}, \quad \theta_{j}=\frac{m_{j}}{2}+\frac{1}{2 p} \\
Y_{0}=\left\{u \in W_{p}^{2}((0, a) ; E(A), E): L_{1} u=0, L_{2} u=0\right\}, \quad f(x)=F(x, 0,0) .
\end{gathered}
$$

Remark 7.1. By Theorem 3.5, the embedding $D^{k} W_{p}^{2}(0, a ; E(A), E) \subset E_{k}$ is continuous and there exist constants $C_{0}$ and $C_{1}$ such that for $w \in Y, W=\left(w_{1}, w_{2}\right), w_{k}=D^{m_{k}} w$, we have

$$
\left\|D^{k} w\right\|_{E_{k}, \infty}=\sup _{x \in[0, a]}\left\|D^{k} w(x)\right\|_{E_{k}} \leq C_{1}\|w\|_{Y}
$$




$$
\|W\|_{0, \infty}=\sup _{x \in[0, a]} \sum_{k=0}^{1}\left\|w_{k}\right\|_{E_{k}} \leq C_{0}\|w\|_{Y} .
$$

By virtue of Theorem 3.5 and Theorem 5.1 , both $C_{0}$ and $C_{1}$ clearly may be chosen independent of $a$.

In what follows, let us assume the following condition:

(C) Let $E$ be a Banach space satisfying the multiplier condition with respect to $p>1$. Suppose there exist $f_{k} \in E_{k}$ such that the operator $A(0, \Phi)$ for $\Phi=\left(f_{1}, f_{2}\right)$ is $R$-positive in $E$.

Theorem 7.2. Assume $(\mathrm{C})$ holds and let the following conditions be satisfied:

(1) $(-1)^{m_{1}} \alpha_{1} \beta_{2}-(-1)^{m_{2}} \alpha_{2} \beta_{1} \neq 0$;

(2) $A(x, U)$ for $U=\left(u_{0}, u_{1}\right), x \in[0, a], u_{k} \in E_{k}$, is a $\varphi$-positive operator in a Banach space $E$ for $0<\varphi \leq \pi$, where the domain of definition $D(A(x, U))$ does not depend on $x, u_{k}$, and $A:[0, a] \times F_{0} \rightarrow B(E(A), E)$ is continuous. Moreover, for each $R>0$ there is a constant $L(R)>0$ such that

$$
\|[A(x, U)-A(x, \bar{U})] u\|_{E} \leq L(R)\|U-\bar{U}\|_{F_{0}}\|A u\|_{E}
$$

for $x \in[0, a], U, \bar{U} \in F_{0}, \bar{U}=\left(\bar{u}_{0}, \bar{u}_{1}\right)$, and $\|U\|_{F_{0}} \leq R,\|\bar{U}\|_{F_{0}} \leq R$

(3) the function $F:[0, a] \times F_{0} \rightarrow E$ is such that $F(\cdot, U)$ is measurable for each $U \in F_{0}$ and $F(t, U)$ is continuous for almost all $x \in[0, a]$. Moreover, for each $R>0$ there is a function $\varphi_{R} \in L_{p}(0, a)$ such that

$$
\|F(x, U)-F(x, \bar{U})\|_{E} \leq \varphi_{R}(x)\|U-\bar{U}\|_{F_{0}}
$$

for almost all $x \in[0, a], U, \bar{U} \in F_{0}$, and $\|U\| \leq R,\|\bar{U}\| \leq R$.

Then there is $a \in\left(0, a_{0}\right)$ such that the problem (2.3)-(2.4) for $|\arg \lambda| \leq \pi-\varphi, 0<\varphi \leq \pi$ and sufficiently large $|\lambda|$ has a unique solution belonging to the space $W_{p}^{2}(0, a ; E(A), E)$.

Proof. We want to solve the problem (2.3)-(2.4) locally by means of maximal regularity of the linear problem (2.1)-(2.2) via the contraction mapping theorem. By virtue of Theorem 5.1, for $|\arg \lambda| \leq \pi-\varphi, 0<\varphi \leq \pi$ and sufficiently large $|\lambda|$, the linear BVP

(7.1) $(L+\lambda) w=-w^{\prime \prime}(x)+A_{\lambda}(0, H) w(x)=f(x), \quad x \in(0, a), \quad L_{1} w=f_{1}, \quad L_{2} w=f_{2}$,

where $H=\left(w\left(x_{0}\right), w^{\prime}\left(x_{0}\right)\right), x_{0} \in(0, a)$, and $L_{k}$ is defined in (2.4), is maximal regular in $X$ and satisfies the estimate

$$
\|w\|_{Y} \leq C\left(\|f\|_{X}+\sum_{k=1}^{2}\left\|f_{k}\right\|_{(E(A), E)_{\theta_{k}, p}}\right),
$$

where $C$ does not depend on $a \in\left(0, a_{0}\right]$. Let $w$ be a solution of the BVP (7.1). Consider a ball

$$
B_{r}=\left\{v \in X: v-w \in Y_{0},\|v-w\|_{Y} \leq r\right\} .
$$

Given $v \in B_{r}$ and $V=\left(v, v^{\prime}\right)$, consider the linear BVP 


$$
\begin{array}{r}
-u^{\prime \prime}(x)+A(0, H) u(x)+\lambda u(x)=F(x, V)+[A(0, H)-A(x, V)] v(x), \quad x \in(0, a), \\
L_{1} u=f_{1}, \quad L_{2} u=f_{2} .
\end{array}
$$

Define a map $Q$ on $B_{r}$ by $Q v=u$, where $u$ is a solution of the problem (7.2). We want to show that $Q\left(B_{r}\right) \subset B_{r}$ and that $L$ is a contraction operator in $Y$, provided $a$ is sufficiently small and $r$ is chosen properly. To this end, by using maximal regularity of the problem (7.1), we obtain from (7.2)

$$
\begin{aligned}
\|Q v-w\|_{Y} & =\|u-w\|_{Y} \\
& \leq C_{0}\left\{\|F(x, V)-F(x, 0)\|_{X}+\|[A(0, H)-A(x, V)] v\|_{X}\right\}
\end{aligned}
$$

Then, by using the assumption (2), we obtain

$$
\begin{aligned}
\| & {[A(0, H)-A(x, V)] v \|_{X} } \\
& \leq \sup _{x \in[0, a]}\left[\|A(0, H)-A(x, H)\|_{B\left(F_{0}, E\right)}+\|A(x, H)-A(x, V)\|_{B\left(F_{0}, E\right)}\right]\|v\|_{Y} \\
& \leq\left[K_{b}+L(R)\|V-H\|_{0, \infty}\right]\left[\|v-w\|_{Y}+\|w\|_{Y}\right] \\
& \leq\left[K_{b}+L(R)\left(C_{1}\|v-w\|_{Y}+\|W-H\|_{0, \infty}\right)\right]\left[\|v-w\|_{Y}+\|w\|_{Y}\right] \\
& \leq\left[K_{b}+L(R)\left(C_{1} r+\|W-H\|_{0, \infty}\right)\right]\left[r+\|w\|_{Y}\right],
\end{aligned}
$$

where $K_{b}=\sup _{x \in[0, a]}\|A(0, H)-A(x, H)\|_{B\left(F_{0}, E\right)}$ and $R=C_{1}+\|W\|_{0, \infty}$ is a fixed number. By assumption (3), we similarly obtain

$$
\begin{aligned}
\|F(x, V)-F(x, 0)\|_{X} & \leq\|F(x, V)-F(x, H)\|_{X}+\|F(x, H)-F(x, 0)\|_{X} \\
& \leq\|\varphi\|_{p}\left[\|V-W\|_{0, \infty}+\|W\|_{0, \infty}\right] \\
& \leq C_{1}\|\varphi\|_{p}\|v-w\|_{Y}+\|W\|_{0, \infty} \\
& \leq\|\varphi\|_{p}\left[C_{1} r+\|W\|_{0, \infty}\right] .
\end{aligned}
$$

Since $r \leq 1$, we have from the above estimates that

$$
\begin{aligned}
\|Q v-w\|_{Y} \leq C_{0}\left\{\left[K_{b}+L(R)\left(C_{1} r+\|W-H\|_{0, \infty}\right)\right]\right. & {\left.\left[r+\|w\|_{Y}\right]\right\} } \\
& +C_{0}\|\varphi\|_{L_{p}}\left[C_{1} r+\|W\|_{0, \infty}\right] .
\end{aligned}
$$

In a similar way, for $v, \bar{v} \in B_{r}, \bar{V}=\left(\bar{v}, \bar{v}^{\prime}\right)$, we obtain

$$
\begin{gathered}
\|Q v-Q \bar{v}\|_{Y} \leq C_{0}\left\{\|F(x, V)-F(x, \bar{V})\|_{X}+\|[A(0, H)-A(x, \bar{V})](v-\bar{v})\|_{X}\right. \\
\left.+\|[A(x, V)-A(x, \bar{V})] \bar{v}\|_{X}\right\} \\
\leq C_{0}\left[\|\varphi\|_{p}\|V-\bar{V}\|_{0, \infty}+\left(K_{b}+L(R)\|H-V\|_{0, \infty}\right)\|v-\bar{v}\|_{Y}\right.
\end{gathered}
$$




$$
\begin{aligned}
& \left.+L(R)\|H-\bar{V}\|_{0, \infty}\|\bar{v}\|_{Y}\right] \\
& \leq C_{0}\left[C_{1}\|\varphi\|_{p}+K_{b}+L(R)\left(\|H-W\|_{0, \infty}+C_{1} r\right)+L(R) C_{1}\left(r+\|w\|_{Y}\right)\right]\|v-\bar{v}\|_{Y} .
\end{aligned}
$$

Now by suitably choosing $r, a \in\left(0, a_{0}\right), f_{k}$, and by using Remark 7.1 and condition $(\mathrm{C})$, we obtain

$$
\|Q v-Q \bar{v}\|_{Y} \leq \delta\|v-\bar{v}\|_{Y}, \quad \text { where } \quad \delta<1,
$$

i.e., $Q$ is a contraction operator. If $a$ is chosen so small, then we have

$$
Q\left(B_{r}\right) \subset B_{r} .
$$

The contraction mapping principle therefore implies the existence of a unique fixed point of $Q$ in $B_{r}$, which is the unique strong solution $u \in Y_{0}$.

\section{NONLOCAL BVPS}

8.1. Elliptic Equations on a Domain with Varying Bound. The Fredholm property of BVPs for elliptic equations in smooth domains was studied in e.g., [2, 5]. In this section we use Theorem 5.1 and Theorem 6.1 in order to establish the maximal $L_{\mathbf{p}}$-regularity for the solution of nonlocal elliptic BVPs on a cylindrical region with varying bound. Let $G \subset \mathbb{R}^{m}, m \geq 2$, be a bounded domain with an $(m-1)$-dimensional boundary $\partial G$ which locally admits rectification. In the domain $\Omega_{t}=[0, b(t)] \times G, t \in[c, d]$, we consider a nonlocal BVP

$$
\begin{aligned}
& (L+\lambda) u:=-D_{x}^{2} u(x, y)-\sum_{k, j=1}^{m} a_{k j}(y) D_{k} D_{j} u(x, y)+a(x, y) D_{x} u(x, y) \\
& +\sum_{j=1}^{m} a_{j}(x, y) D_{j} u(x, y)+a_{0}(x, y) u(x, y)+\lambda u(x, y)=f(x, y), \\
& L_{t k} u:=\alpha_{k} \frac{\partial^{m_{k}}}{\partial x^{m_{k}}} u(0, y)+\beta_{k} \frac{\partial^{m_{k}}}{\partial x^{m_{k}}} u(1, y)+\sum_{j=1}^{N_{k}} \delta_{k j} \frac{\partial^{m_{k}}}{\partial x^{m_{k}}} u\left(x_{k j}, y\right) \\
& \quad+\sum_{j=-1}^{M_{k}} T_{k j} u\left(x_{k j 0}, y\right)=f_{k}(y), \quad k \in\{1,2\}, \quad y \in G, \\
& L_{0} u:=\sum_{j=1}^{m} c_{j}\left(y^{\prime}\right) \frac{\partial}{\partial y_{j}} u\left(x, y^{\prime}\right)+c_{0}\left(y^{\prime}\right) u\left(x, y^{\prime}\right)=0, \quad x \in(0,1), \quad y^{\prime} \in \partial G,
\end{aligned}
$$

where $D_{x}=\frac{\partial}{\partial x}, D_{j}=-i \frac{\partial}{\partial y_{j}}, D_{y}=\left(D_{1}, \ldots, D_{m}\right), m_{k} \in\{0,1\}, \alpha_{k}, \beta_{k}, \delta_{k j}$ are complexvalued continuous functions of $t \in[c, d], r=$ ord $L_{0}, y=\left(y_{1}, \ldots, y_{m}\right), x_{k j} \in(0,1)$, $x_{k j 0} \in[0,1]$, and $T_{k j}=T_{k j}(t)$ are, in general, unbounded operators in $L_{p}(G)$.

Theorem 8.1. Let the following conditions be satisfied: 
(1) $a_{k j} \in C(\bar{G}), a, a_{j}, a_{0} \in C^{1}(\bar{G}), c_{0} \in C(\bar{G}), \partial G \in C^{2}$;

(2) $\sum_{j=1}^{m} c_{j}\left(y^{\prime}\right) \sigma_{j} \neq 0, y^{\prime} \in \partial G, \sigma \in \mathbb{R}^{m}$ is normal to $\partial G, c_{j}, c_{0} \in C^{1}(\bar{G})$ for $r=1$ and $c_{0}\left(y^{\prime}\right) \neq 0, y^{\prime} \in \partial G$ for $r=0$;

(3) for $y \in G, \sigma \in \mathbb{R}^{m}, \arg \lambda=\pi,|\sigma|+|\lambda| \neq 0, \lambda+\sum_{k, j=1}^{m} a_{k j}(y) \sigma_{k} \sigma_{j} \neq 0$;

(4) for the tangent vector $\sigma^{\prime}$ and a normal vector $\sigma$ to $\partial G$ at the point $y^{\prime} \in \partial G$, the boundary value problem

$$
\begin{gathered}
{\left[\lambda+\sum_{k, j=1}^{m} a_{k j}\left(y^{\prime}\right)\left(\sigma_{k}^{\prime}-i \sigma_{j} \frac{d}{d \xi}\right)\left(\sigma_{k}^{\prime}-i \sigma_{j} \frac{d}{d \xi}\right)\right] u(\xi)=0, \quad \xi>0, \quad \lambda \leq 0,} \\
\left.\sum_{j=1}^{m} c_{j}\left(y^{\prime}\right)\left(\sigma_{k}^{\prime}-i \sigma_{j} \frac{d}{d \xi}\right) u(\xi)\right|_{\xi=0}=h
\end{gathered}
$$

(and for $r=0$, the problem generated by the same equations with $u(0)=h$ ) has one and only one solution which, including all of its derivatives, tends to zero as $\xi \rightarrow \infty$ for any numbers $h \in \mathbb{C}$;

(5) $(-1)^{m_{1}} \alpha_{1} \beta_{2}-(-1)^{m_{2}} \alpha_{2} \beta_{1} \neq 0$ for $t \in[c, d]$;

(6) if $m_{k}=0$, then $T_{k j}=0$; if $m_{k}=1$, then for any $\varepsilon>0$ there is $c(\varepsilon)$ such that for all $u \in B_{\mathbf{p}}^{2-\frac{1}{p_{1}}}\left(G ; L_{0} u=0, r<2-m_{k}-2 / p_{2}\right)$,

$$
\left\|T_{k j} u\right\|_{B_{\mathbf{p}}^{1-\frac{1}{p_{1}}}(G)} \leq \varepsilon\|u\|_{B_{\mathbf{p}}^{2-\frac{1}{p_{1}}}(G)}+c(\varepsilon)\|u\|_{L_{p_{1}}(G)},
$$

where $p_{2} \in(1, \infty) \backslash\{2\}$, or $p_{2}=2$ and $r \neq 1$.

Then, for $f \in L_{\mathbf{p}}\left(\Omega_{t}\right), \mathbf{p}=\left(p_{1}, p_{2}\right), 0<p_{1}, p_{2}<\infty, f_{k} \in B_{\mathbf{p}}^{2-m_{k}-\frac{1}{p_{1}}}(G)$ and $|\arg \lambda|=\pi$ and sufficiently large $|\lambda|$, the problem (8.1)-(8.3) has a unique solution that belongs to the space $W_{\mathbf{p}}^{2}\left(\Omega_{t}\right)$, and coercive uniformity with respect to the parameters $t$ and $\lambda$ holds, i.e.,

$$
\|u\|_{W_{\mathbf{p}}^{2}\left(\Omega_{t}\right)} \leq C\left[\|(L+\lambda) u\|_{L_{\mathbf{p}}\left(\Omega_{t}\right)}+\sum_{k=1}^{2}\left\|L_{k} u\right\|_{B_{\mathbf{p}}^{2-m_{k}-\frac{1}{p_{1}}(G)}}+\|u\|_{L_{\mathbf{p}}\left(\Omega_{t}\right)}\right]
$$

is satisfied for the solution of the problem (8.1)-(8.3). Moreover, the operator

$$
u \rightarrow Q(t) u=\left\{L u, L_{t 1} u, L_{t 2} u\right\}
$$

from $W_{\mathbf{p}}^{2}\left(\Omega_{t} ; L_{0} u=0\right)$ into

$$
L_{\mathbf{p}}\left(\Omega_{t}\right) \times \prod_{k=1}^{2} B_{\mathbf{p}}^{2-m_{k}-\frac{1}{p_{1}}}\left(G, L_{0} u=0, r<2-m_{k}-2 / p_{2}\right)
$$

is bounded and Fredholm.

Proof. Let $E=L_{p_{1}}(G)$. Consider the operator $A$ defined by

$$
D(A)=W_{p_{1}}^{2}\left(G ; L_{0} u=0\right), \quad A u=-\sum_{k, j=1}^{m} a_{k j}(y) D_{k} D_{j} u(x, y) .
$$


For $x \in[0, b]$, also consider the operators

$$
B_{1}(x) u=a(x, y) u(x, y), \quad B_{2}(x) u=\sum_{j=1}^{m} a_{j}(x, y) D_{j} u(x, y)+a_{0}(x, y) u(x, y) .
$$

Let us apply Theorem 6.1 to the problem (8.1)-(8.3). Using [5, Theorem 8.2], the operator $A+\mu I$ is $R$-positive in $L_{p_{1}}$ for sufficiently large $\mu \geq 0$. Moreover, it is known that the embedding $W_{p_{1}}^{2}(G) \subset L_{p_{1}}(G)$ is compact (see, e.g., Triebel [10, Theorem 3.2.5]). Then due to the positivity of $A+\mu I$ in $L_{p_{1}}(G)$, we obtain that $(A+\mu I)^{-1} \in \sigma_{\infty}\left(L_{p_{1}}(G)\right)$. Therefore, condition (1) of Theorem 6.1 is fulfilled. Condition (2) of Theorem 6.1 coincides with condition (5). By virtue of condition (1) of Theorem 8.1, the operators $B_{1}(x)$ in $L_{p_{1}}(G)$ and $B_{2}(x)$ from $W_{p_{1}}^{1}(G)$ to $L_{p_{1}}(G)$ are bounded. Using $[10, \S 4.3 .3]$ and in view of the embedding between Sobolev and Besov spaces [4, §18, Theorem 18.9], we have

$$
(E(A), E)_{\frac{1}{2}, 1}=\left(W_{p_{1}}^{2}\left(G, L_{0}\right), L_{p_{1}}(G)\right)_{\frac{1}{2}, 1}=B_{p_{1}, 1}^{1}\left(G, L_{0}, r=0\right) \subset W_{p_{1}}^{1}(G) .
$$

Therefore, the operator $B_{1}(x)$ from $W_{p_{1}}^{1}(G)$ into $L_{p_{1}}(G)$ and, consequently, from $(E(A), E)_{\frac{1}{2}, 1}$ into $L_{p_{1}}(G)$, is compact. Then we obtain that the operator $B_{1}(x)$ satisfies condition (3) of Theorem 6.1. In a similar way, we prove that the operator $B_{2}(x)$ satisfies condition (3) of Theorem 6.1, too. Moreover, using interpolation properties of Sobolev spaces (see, e.g., [10, §4]), it is easy to see that condition (4) of Theorem 6.1 is also fulfilled.

8.2. Infinite Systems of Nonlinear Differential Equations. Consider a BVP for an infinite system

$$
\begin{gathered}
-u_{m}^{\prime \prime}(x)+\left[A_{m}\left(x, u, u^{\prime}\right)+\lambda\right] u_{m}(x)=F_{m}\left(x, u, u^{\prime}\right), \quad x \in(0, a), \quad m \in \mathbb{N}, \\
\alpha_{k} D^{m_{k}} u_{m}(0)+\beta_{k} D^{m_{k}} u_{m}(a)+\sum_{i=1}^{N_{k}} \delta_{k i} D^{m_{k}} u_{m}\left(\delta_{k i}\right)=f_{k m}, \quad k \in\{1,2\},
\end{gathered}
$$

where $u=\left\{u_{m}\right\}$ and $u^{\prime}=\left\{u_{m}^{\prime}\right\}$. Let

$$
\begin{gathered}
A\left(x, u, u^{\prime}\right)=\left\{A_{m}\left(x, u, u^{\prime}\right)\right\}, \quad A=A(0,0,0), \quad u=\left\{u_{m}\right\} \in \ell_{q}, \\
D(A)=\ell_{q}^{s}:=\left\{u \in \ell_{q}:\|u\|_{l_{q}^{s}}:=\left(\sum_{m=1}^{\infty}\left|2^{m s} u_{m}\right|^{q}\right)^{\frac{1}{q}}<\infty\right\}, \quad s>0, \quad q \in(1, \infty), \\
A u=\sum_{m=1}^{\infty} 2^{m s} u_{m}, \quad \eta_{k}=\frac{k+1 / p}{2}, \quad F_{0}=\prod_{k=0}^{1} \ell_{p}^{s\left(1-\eta_{k}\right)} .
\end{gathered}
$$

From Theorem 7.2 by using $[10, \S 1.18 .2]$, we obtain the following result.

Theorem 8.2. Let the following conditions be satisfied:

(1) $(-1)^{m_{1}} \alpha_{1} \beta_{2}-(-1)^{m_{2}} \alpha_{2} \beta_{1} \neq 0$; 
(2) the functions $A_{m}(x, U)$ and $F_{m}(x, U)$ are continuously differentiable on $\left[0, a_{0}\right]$ for $U \in F_{0}$.

Then there is $a \in\left(0, a_{0}\right)$ such that the problem (8.4)-(8.5) for $|\arg \lambda| \leq \pi-\varphi, 0<\varphi \leq \pi$, and sufficiently large $|\lambda|$ has a unique solution belonging to the space $W_{p}^{2}\left(0, a ; \ell_{q}^{s}, \ell_{q}\right)$.

Remark 8.3. It should be noted that the assertion of Theorem 8.2 is valid for all finite systems satisfying the conditions of Theorem 8.2.

\section{REFERENCES}

[1] Ravi P. Agarwal, Martin Bohner, and Veli B. Shakhmurov. Maximal regular boundary value problems in Banach-valued weighted space. Boundary Value Problems, 1(1):9-42, 2005.

[2] S. Agmon and L. Nirenberg. Properties of solutions of ordinary differential equations in Banach space. Comm. Pure Appl. Math., 16:121-239, 1963.

[3] Herbert Amann. Linear and quasilinear parabolic problems. Vol. I, volume 89 of Monographs in Mathematics. Birkhäuser Boston Inc., Boston, MA, 1995.

[4] O. V. Besov, V. P. Il'in, and S. M. Nikol'ski1. Integralnye predstavleniya funktsii i teoremy vlozheniya. Izdat. "Nauka", Moscow, 1975.

[5] Robert Denk, Matthias Hieber, and Jan Prüss. $\mathcal{R}$-boundedness, Fourier multipliers and problems of elliptic and parabolic type. Mem. Amer. Math. Soc., 166(788):viii+114, 2003.

[6] Robert Haller, Horst Heck, and André Noll. Mikhlin's theorem for operator-valued Fourier multipliers in $n$ variables. Math. Nachr., 244:110-130, 2002.

[7] S. G. Kreı̆n. Linear differential equations in Banach space. American Mathematical Society, Providence, R.I., 1971.

[8] J.-L. Lions and J. Peetre. Sur une classe d'espaces d'interpolation. Inst. Hautes Études Sci. Publ. Math., 19:5-68, 1964.

[9] Veli B. Shakhmurov. Coercive boundary value problems for regular degenerate differential-operator equations. J. Math. Anal. Appl., 292(2):605-620, 2004.

[10] Hans Triebel. Interpolation theory, function spaces, differential operators, volume 18 of NorthHolland Mathematical Library. North-Holland Publishing Co., Amsterdam, 1978.

[11] Lutz Weis. Operator-valued Fourier multiplier theorems and maximal $L_{p^{-}}$-regularity. Math. Ann., 319(4):735-758, 2001.

[12] Sasun Yakubov and Yakov Yakubov. Differential-operator equations, volume 103 of Chapman 83 Hall/CRC Monographs and Surveys in Pure and Applied Mathematics. Chapman \& Hall/CRC, Boca Raton, FL, 2000.

Florida Institute of Technology, Department of Mathematical Science, Melbourne, FL 32901-6975, U.S.A.

E-mail address: agarwal@fit.edu

University of Missouri-Rolla, Department of Mathematics and Statistics, Rolla, MO 65409-0020, U.S.A.

E-mail address: bohner@umr.edu

Istanbul University, Engineering Faculty, Department of Electrical-Electronics Engineering, Avcilar, 34850 Istanbul, Turkey

E-mail address: sahmurov@istanbul.edu.tr 